\title{
Article
}

\section{Numerical Simulation of Particle Dispersion in Flow between Coaxial Cylinders under Unsteady Flow Conditions}

\author{
Koji MASUDA ${ }^{*}$, , Hiroshi SUZUKI ${ }^{* *}$, Yoshiyuki KOMODA ${ }^{* *}$, and Ruri HIDEMA ${ }^{* * *}$ \\ "Department of Applied Chemistry, Kobe City College of Technology \\ 8-3, Gakuenhigashi-machi, Nishi-ku, Kobe-shi, Hyogo 651-2194, Japan \\ ${ }^{* *}$ Department of Chemical Science and Engineering, Kobe University \\ 1-1, Rokkodai-cho, Nada-ku, Kobe-shi, Hyogo 657-8501, Japan \\ ${ }^{* * *}$ Organization of Advanced Science and Technology, Kobe University \\ 1-1, Rokkodai-cho, Nada-ku, Kobe-shi, Hyogo 657-8501, Japan \\ (Received : May 1, 2015)
}

\begin{abstract}
Two-dimensional numerical computations of unsteady flow of a suspension between two coaxial cylinders are performed. We investigate the dispersion characteristics based on a novel thixotropy model. The diameter of particles, solid volume fraction, and viscosity of dispersing media are set to $2.5 \mu \mathrm{m}, 0.15$, and $195 \mathrm{~Pa} \cdot \mathrm{s}$, respectively. The shear rate is suddenly changed from $0.1 \mathrm{~s}^{-1}$ to $1 \mathrm{~s}^{-1}$. For model validation, the numerical results are compared with previously reported experimental results. The mean number of particles in a cluster increases slightly shortly after the shear rate change, reaches a maximum, and subsequently decreases, finally attaining a steady state at $40 \mathrm{~s}$ after the shear rate change. Our model better expresses the time variation of the dispersion characteristics than the previous model. After the validation, the effect of bonding energy on the time-variation characteristics is investigated. The bonding energy is varied as $2.7 \times 10^{-13}, 2.7 \times 10^{-12}$, and $2.7 \times 10^{-11} \mathrm{~J}$. We observe that the asymptotic values of the mean number of particles in a cluster depend on the bonding energy, but this relationship is not linear. From our results, we posit the existence of a certain critical bonding energy with regard to the dispersion characteristics.
\end{abstract}

Key Words: Numerical simulation / Particle dispersion / Unsteady flow / Kneading extruder / Polymer composites

\section{INTRODUCTION}

Polymer composites containing fine particles (e.g., silica) have attracted considerable attention because such composites exhibit a wide range of functionalities with applications ranging from bone tissue engineering to aerospace components. These composites are made from suspensions that disperse particles in the molten polymer. However, the dispersion of fine particles in the polymer is not an easy process to achieve. The fine particles have a high specific surface area, and consequently, the interparticle energy resulting from surface energy becomes relatively large. Thus, the fine particles easily aggregate with each other and form larger clusters. Since the functionality of the polymer composites depends on the particle dispersion, it is essential to control the dispersion characteristics of particles in molten polymer.

In order to disperse particles in polymers, a kneading extruder with rotors is often used. In such extruders, high

$\dagger$ masuda@kobe-kosen.ac.jp shear stresses are applied to break up the clusters with the use of rotors. However, the shear stresses are timevarying and space-varying (different for different locations) in the extruder. Consequently, the particle dispersion in the polymer composites is nonuniform over both space and time. Therefore, it is very difficult to determine the dispersion characteristics of the particles in such extruders via experimental studies.

The particle behaviors in solid-liquid suspensions are affected by several internal and external factors. Smoluchowski ${ }^{1)}$, Swift and Friedlander ${ }^{2}$, and Higashitani et al. $^{3-5)}$ have proposed the coagulation model of fine particles in suspensions. On the other hand, Usui ${ }^{6}$ has established a thixotropy model considering the shear breakup of clusters in suspensions; he has also suggested a method for predicting the rate of change in the cluster size and bulk suspension viscosity. This model has been applied and validated for several polymer composite systems. ${ }^{7,8)}$ The model suggested by Usui can estimate the mean number of particles in a cluster. Since the existence of large clusters severely affects the function of the polymer composites, Hasegawa et al..$^{9,10)}$ 
have further developed Usui's model for estimating the cluster size distributions; however, their model can estimate particle dispersion characteristics only for a simple shear flow. In other previous studies ${ }^{11,12)}$, a novel thixotropy model was developed for estimating the cluster size under the conditions of unsteady nonuniform flow. With this model, the time-variation cluster size distributions can be locally obtained by considering the local balance of Brownian and shear coagulations and shear break-up of clusters. Masuda et al. ${ }^{11)}$ have confirmed that this model is sufficiently accurate for steady nonuniform flow by comparing the modeling results with the experimental data obtained for a flow with a backward-facing step. However, accuracy regarding the time-dependency of the particle dispersion characteristics as estimated by the model has not thus far been confirmed.

In this study, we apply this thixotropy model to simulate the unsteady dispersion characteristics of fine particles in a flow (of the suspension of interest) between coaxial cylinders with the inner cylinder undergoing rotation. Our attempt is the first step toward investigating particle dispersion characteristics in an extruder with rotors. The flow geometries for the abovementioned conditions have been examined by several researchers investigating the Taylor instability of Newtonian fluid flow ${ }^{13)}$, unsteady particle coagulation ${ }^{14)}$, and the shear banding phenomena of non-Newtonian fluid flow. ${ }^{15)}$ In the present study, we focus on the unsteady dispersion behavior when the shear rate is suddenly changed from $0.1 \mathrm{~s}^{-1}$ to $1 \mathrm{~s}^{-1}$ before the flow is fully developed as a steady Couette flow. In such a situation, the fine particle clusters break up nonuniformly in space and time.

We first compare our simulations with the experimental data obtained by Komoda et al. ${ }^{16)}$ in order to confirm the accuracy of the model suggested by Masuda et al. ${ }^{11)}$ Next, we investigate the effect of interparticle bonding energy (which characterizes the shear-breakup of the clusters of particles) on the cluster size. From the results of this investigation, we elucidate the mechanism of particle dispersion under an unsteady flow.

\section{NUMERICAL METHODS}

Figure 1 shows the computational domain considered in this study. The inner cylinder with a diameter of $22.8 \mathrm{~mm}$ rotates along the anticlockwise direction with a surface tangential velocity, $u_{\text {wall }}\left[\mathrm{m} \cdot \mathrm{s}^{-1}\right]$, while the outer cylinder with a diameter of $38 \mathrm{~mm}$ is fixed. The test suspension fills the space between the coaxial cylinders. The parameters $r$ [m] and $\theta$ [rad] are defined as the radial and circumferential coordinates, respectively, as shown in the figure.

The computations in our study are performed with twodimensional continuity and momentum equations for fluid flow, which are expressed as

$$
\begin{aligned}
& \frac{1}{r} \frac{\partial\left(r u_{r}\right)}{\partial r}+\frac{1}{r} \frac{\partial u_{\theta}}{\partial \theta}=0 \\
& \rho\left(\frac{\partial u_{r}}{\partial t}+u_{r} \frac{\partial u_{r}}{\partial r}+\frac{u_{\theta}}{r} \frac{\partial u_{r}}{\partial \theta}-\frac{u_{\theta}^{2}}{r}\right) \\
& =-\frac{\partial P}{\partial r}+\left[\frac{1}{r} \frac{\partial}{\partial r}\left(r \tau_{r r}\right)+\frac{1}{r} \frac{\partial \tau_{r \theta}}{\partial \theta}-\frac{\tau_{\theta \theta}}{r}\right] \\
& \rho\left(\frac{\partial u_{\theta}}{\partial t}+u_{r} \frac{\partial u_{\theta}}{\partial r}+\frac{u_{\theta}}{r} \frac{\partial u_{\theta}}{\partial \theta}+\frac{u_{r} u_{\theta}}{r}\right) \\
& =-\frac{1}{r} \frac{\partial P}{\partial \theta}+\left[\frac{1}{r^{2}} \frac{\partial}{\partial r}\left(r^{2} \tau_{r \theta}\right)+\frac{1}{r} \frac{\tau_{\theta \theta}}{\partial \theta}\right]
\end{aligned}
$$

Here, $u_{r}\left[\mathrm{~m} \cdot \mathrm{s}^{-1}\right]$ and $u_{\theta}\left[\mathrm{m} \cdot \mathrm{s}^{-1}\right]$ represent the radial and circumferential velocity components, respectively, while $t[\mathrm{~s}], P[\mathrm{~Pa}]$, and $\rho\left[\mathrm{kg} \cdot \mathrm{m}^{-3}\right]$ denote the time, pressure, and suspension density, respectively. The stress components, $\tau_{r r}$ $[\mathrm{Pa}], \tau_{r \theta}[\mathrm{Pa}]$, and $\tau_{\theta \theta}[\mathrm{Pa}]$, are computed using the following equations. Here, $\eta[\mathrm{Pa} \cdot \mathrm{s}]$ denotes the suspension viscosity. In the present study, the suspension viscosity is estimated using the following model proposed by Simha. ${ }^{17)}$

$$
\frac{\eta}{\eta_{0}}=1+2.5 \lambda \phi
$$

Here, $\eta_{0}[\mathrm{~Pa} \cdot \mathrm{s}]$ and $\mathrm{f}[-]$ denote the viscosity of the dispersed media and the apparent solid volume fraction, respectively. Further, $\lambda[-]$ denotes the function of the computational domain cell ratio, and it is related to the parameter $\xi[-]$ as follows.

$$
\lambda=\frac{4\left(1-\xi^{7}\right)}{4\left(1+\xi^{10}\right)-25 \xi^{3}\left(1+\xi^{4}\right)+42 \xi^{5}}
$$

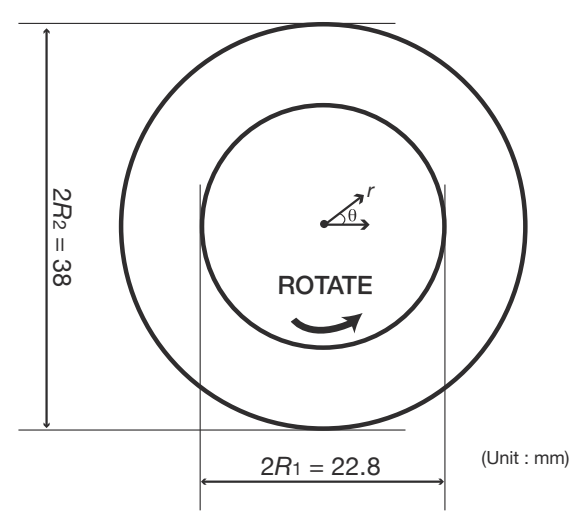

Fig. 1. Computational domain used in our simulation. 
The parameter $\xi$ is estimated from the apparent volume fraction by means of the following equation.

$$
\xi^{3}=\frac{\phi}{f^{3}\left[1-\left(\phi^{\frac{1}{3}} / f\right)\right]^{3}}
$$

Here, $f^{3}[-]$ represents the supposed volume fraction of the total cell. When $\phi$ is equal to the maximum packing fraction, $\phi_{\mathrm{p}, \max }[-], \xi$ becomes unity and $\phi_{\mathrm{p}, \max }=f^{3} / 8$. By using Eq. (6), we calculate the apparent volume fraction from the cluster size distribution.

$$
\phi=\sum_{k} \frac{\pi}{6} d_{k}^{3} n_{k}
$$

Here, $d_{k}[\mathrm{~m}]$ and $n_{k}\left[\mathrm{~m}^{-3}\right]$ denote the cluster size and concentration of a cluster composed of $k$ particles, respectively.

In the present study, the apparent solid volume fraction is calculated via the thixotropy model considering the convections suggested by Masuda et al.. ${ }^{11)}$ At first, the cluster distribution at each computational cell is computed by means of the following equation.

$$
\begin{aligned}
& \frac{\partial n_{k}}{\partial t}+u_{r} \frac{\partial\left(n_{k}\right)}{\partial r}+\frac{u_{\theta}}{r} \frac{\partial n_{k}}{\partial \theta}= \\
& \frac{1}{2} \alpha_{\mathrm{b}} \sum_{i=1, i+j=k}^{i=k-1} \frac{2 k_{\mathrm{b}} T}{3 \eta_{0}}\left(r_{i}+r_{j}\right)\left(\frac{1}{r_{i}}+\frac{1}{r_{j}}\right) n_{i} n_{j} \\
& \quad-\alpha_{\mathrm{b}} \sum_{i=1}^{\infty} \frac{2 k_{\mathrm{b}} T}{3 \eta_{0}}\left(r_{i}+r_{k}\right)\left(\frac{1}{r_{i}}+\frac{1}{r_{k}}\right) n_{i} n_{k} \\
& +\frac{1}{2} \alpha_{\mathrm{s}} \sum_{i=1, i+j=k}^{i=j-1} \frac{4 \dot{\gamma}}{3}\left(r_{i}+r_{j}\right)^{3} n_{i} n_{j} \\
& -\frac{3}{4} \frac{\pi \cdot \alpha_{\mathrm{s}} \sum_{i=1}^{\infty}}{F_{0} N_{\mathrm{b}}} \frac{4 \dot{\gamma}}{3}\left(r_{i}+r_{k}\right)^{3} n_{i} n_{k} \\
& +\frac{3}{(k-1)}\left(\frac{k}{1-\varepsilon}-1\right) n_{k} \\
& +\frac{3}{4} \frac{\dot{\gamma}^{2}}{F_{0} N_{\mathrm{b}}} \frac{\eta \cdot d_{0}^{3}}{(2 k-1)}\left(\frac{2 k}{1-\varepsilon}-1\right) n_{2 k} \\
& +\frac{3}{4} \frac{\pi \cdot d_{0}^{3}}{F_{0} N_{\mathrm{b}}} \frac{\dot{\gamma}^{2}}{(2 k-2)}\left(\frac{2 k-1}{1-\varepsilon}-1\right) d_{0}^{3} \\
& \left.\frac{2 k-1}{1-\varepsilon}-1\right) n_{2 k+1}
\end{aligned}
$$

Here, $r_{i}[\mathrm{~m}], k_{\mathrm{b}}\left[\mathrm{J} \cdot \mathrm{K}^{-1}\right], T[\mathrm{~K}], \varepsilon[-], d_{0}[\mathrm{~m}]$, and $\dot{\gamma}\left[\mathrm{s}^{-1}\right]$ denote the cluster radius composed of $i$ particles, Boltzmann constant, absolute temperature of the fluid, void fraction of particles in a cluster, diameter of the primary particle, and shear rate applied to the suspension, respectively. Parameters $\alpha_{\mathrm{b}}(=0.58)$ [-] and $\alpha_{\mathrm{s}}(=0.60)[-]$ denote the kinetic constants for Brownian coagulation and shear coagulation, respectively, as reported by Higashitani et al. Further, parameters, $F_{0}[\mathrm{~J}]$ and $N_{\mathrm{b}}[-]$ denote the bonding energy between particles and the number of bonds to be broken when the cluster is divided into two smaller similarly sized clusters, respectively. Parameter $N_{\mathrm{b}}$ is estimated as follows.

$$
N_{\mathrm{b}}=\frac{k d_{0}}{2 d_{k}}
$$

The cluster size, $d_{\mathrm{k}}[\mathrm{m}]$, is calculated as follows.

$$
d_{k}=d_{0}\left(\frac{k}{1-\varepsilon}\right)^{\frac{1}{3}}
$$

In order to estimate the void fraction in a cluster, Usui has proposed the following equation.

$$
\varepsilon=\varepsilon_{\max }\left(1-k^{-0.4}\right)
$$

Here, $\varepsilon_{\max }$ denotes the maximum void fraction estimated from the original solid fraction, $\phi_{0}[-]$, and the maximum solid fraction, $\phi_{\mathrm{p}, \max }[-]$, as follows.

$$
\varepsilon_{\max }=1-\frac{\phi_{0}}{\phi_{\mathrm{p}, \max }}
$$

From these equations, $n_{k}$ can be calculated when the bonding energy, $F_{0}$, is given. Parameter $n_{k}$ yields the local apparent solid fraction, $\phi$, via Eq. (6). Thus, the local viscosity can be obtained by using Simha's model. As per Usui, the bonding energy can be obtained when the suspension viscosity at a certain shear rate can be obtained at a steady state by rheological measurements. Using Usui's relation described above, Hasegawa et al. ${ }^{9)}$ have reported the bonding energy between silica particles in ethylene-methyl methacrylate (EMMA) copolymer to be $2.7 \times 10^{-12} \mathrm{~J}$.

In order to validate the present numerical method, we first compare our simulations with the experimental results for a silica suspension in EMMA, as reported by Komoda et al. In the computation, the shear rate is suddenly changed from $0.1 \mathrm{~s}^{-1}$ to $1 \mathrm{~s}^{-1}$. Before the sudden change, the velocity field is fully developed and the cluster size distributions experimentally obtained at the steady state of $0.1 \mathrm{~s}^{-1}$ are uniformly added to each cell as the initial conditions for the computation.

Particle size $d_{0}$, solid volume fraction $\phi_{0}$, and viscosity of dispersing media are set at $2.5 \mu \mathrm{m}, 0.15$, and $195 \mathrm{~Pa} \cdot \mathrm{s}$, respectively, for EMMA. The dispersing media densities are set to $1000 \mathrm{~kg} \cdot \mathrm{m}^{-3}$.

After model validation, we discuss the effect of bonding energy on the time-variation characteristics. The bonding energy is varied over three steps as $2.7 \times 10^{-13}, 2.7 \times 10^{-12}$, 
and $2.7 \times 10^{-11} \mathrm{~J}$, while the solid volume fraction is fixed to 0.15 . The shear rate is suddenly changed from $0.1 \mathrm{~s}^{-1}$ to $1 \mathrm{~s}^{-1}$.

\section{RESULTS AND DISCUSSION}

Figure 2 shows the time dependency of the overall mean number of particles in a cluster, $k_{\mathrm{m}}[-]$. The solid circles represent the experimental data obtained by Komoda et al. and the solid line represents our study result. As regards our study, the figure shows the results obtained at $\left(r-R_{1}\right) /\left(R_{2}-R_{1}\right)$ $=0.36$, where $R_{1}$ and $R_{2}$ denote the radii of the inner and outer cylinders, respectively. This ratio aids in defining the region of flow between the two cylinders; the smaller is the ratio, the closer is the region of interest to the inner cylinder wall. The bonding energy between particles, $F_{0}$, is $2.7 \times 10^{-12} \mathrm{~J}$. The broken line in the figure denotes the results of the numerical analysis performed by Hasegawa et al. ${ }^{9)}$ under the assumption that the shear rate is uniformly added to the fluid. As regards the results of the present study, the mean number of particles in a cluster increases slightly (with respect to the initial value) shortly after the shear rate change, reaches a maximum, and subsequently decreases and attains a steady state at $t=40 \mathrm{~s}$.

On the other hand, as regards the numerical results obtained by Hasegawa et al., ${ }^{9)}$ the mean number of particles in a cluster more slowly decreases (there is no initial increase as in the case of our result) and continues to gradually decrease after $40 \mathrm{~s}$. This difference in the time dependency can be attributed to the assumption (as regards the results obtained by Hasegawa et al.) that the shear rate is added uniformly. Thus, the present model better expresses the time variation of the dispersion characteristics than that obtained by Hasegawa et al..$^{9}$

In our study, when the rotation speed was increased, a large difference in circumferential velocity was generated along the radial direction. Subsequently, the clusters collided with each other, and large clusters were temporarily formed immediately after the shear rate change. These impermanent large clusters were broken up owing to the application of large shear, and subsequently, the mean number of particles in a cluster decreased gradually.

We remark here that Hasegawa's model ${ }^{9)}$ that considers the uniform shear field does not sufficiently accurately express the flow with sudden shear rate change in comparison with the present calculation. On the other hand, in the experimental study by Komoda et al. ${ }^{16}$, a small shear was applied as the initial condition, and subsequently, the shear rate was varied drastically. The present model assumes nonuniform shear flow, and consequently, the results indicate good conformity of the model with the experimental results.

Figure 3 shows the time variations of the velocity profiles at $t=0.01,0.5,2$, and $100 \mathrm{~s}$. We observe that the velocity near the inner cylinder steeply increases immediately after the shear rate change. At this point, the local shear rate is larger than the mean shear rate of $1 \mathrm{~s}^{-1}$. On the other hand, the local shear rate is very small near the outer cylindrical region. Thus, the shear rate is not uniform immediately after the shear rate change. The velocity profile is nearly completely developed at $t=2 \mathrm{~s}$.

The bonding energy corresponding to the results in this figure is $2.7 \times 10^{-12} \mathrm{~J}$. For other bonding energies, the velocity profile and time required for velocity development did not differ significantly. Figure 4 shows the time dependence of the circumferential velocities at $\left(r-R_{1}\right) /\left(R_{2}-R_{1}\right)=0.03,0.36$, 0.62 , and 0.91 . In the figure, the circumferential velocities are normalized by steady-state velocities at each point. These results indicate that the velocity develops in sequence from near the inner cylinder. The velocity profile is developed fully

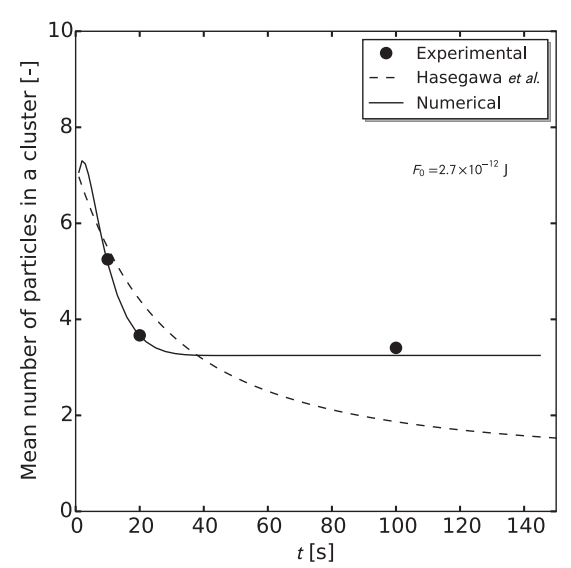

Fig. 2. Time dependence of the mean number of particles in a cluster $\left(k_{\mathrm{m}}\right)$.

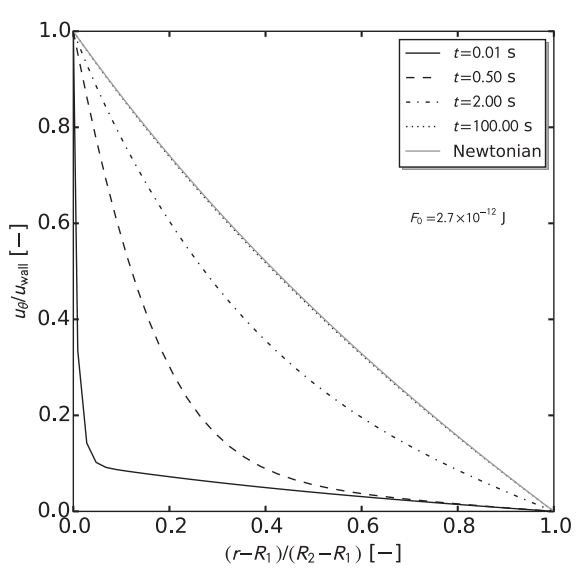

Fig. 3. Velocity profile obtained using our model. 
at $t=80 \mathrm{~s}$. The circumferential velocities exhibit a temporary decrease for $\left(r-R_{1}\right) /\left(R_{2}-R_{1}\right)=0.36,0.62$, and 0.91 . These velocities subsequently increase to finally reach a steady state. The velocities at $\left(r-R_{1}\right) /\left(R_{2}-R_{1}\right)=0.36$ and 0.62 take on minimum values at $t=10 \mathrm{~s}$, and that at $\left(r-R_{1}\right) /\left(R_{2}-R_{1}\right)=0.91$ takes on a minimum value at $t=20 \mathrm{~s}$.

Figure 5 shows the time dependence of viscosity at $\left(r-R_{1}\right) /$ $\left(R_{2}-R_{1}\right)=0.03$ and 0.36 along with the experimental results obtained by Hasegawa." As regards the experimental results, the viscosity slightly increases (with respect to the initial value) immediately after increase in the shear rate. Similarly, the viscosity at $\left(r-R_{1}\right) /\left(R_{2}-R_{1}\right)=0.36$ increases after the shear rate change. The experimental viscosity and numerical viscosity obtained with the present model were $390 \mathrm{~Pa} \cdot \mathrm{s}$ and $550 \mathrm{~Pa} \cdot \mathrm{s}$, respectively, at $t=100 \mathrm{~s}$. Further, the shear rate at steady state was $1 \mathrm{~s}^{-1}$. Upon comparing Figs. 2 and 5, we observe that the time variation characteristics of the viscosity

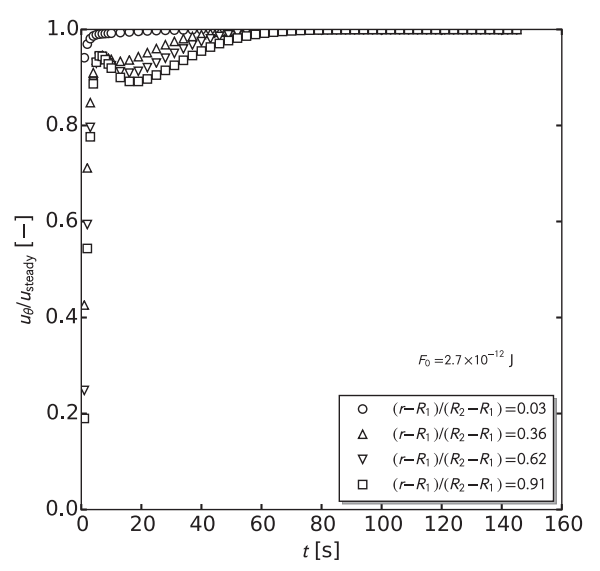

Fig. 4. Variation in circumferential velocity as a function of time as per our model.

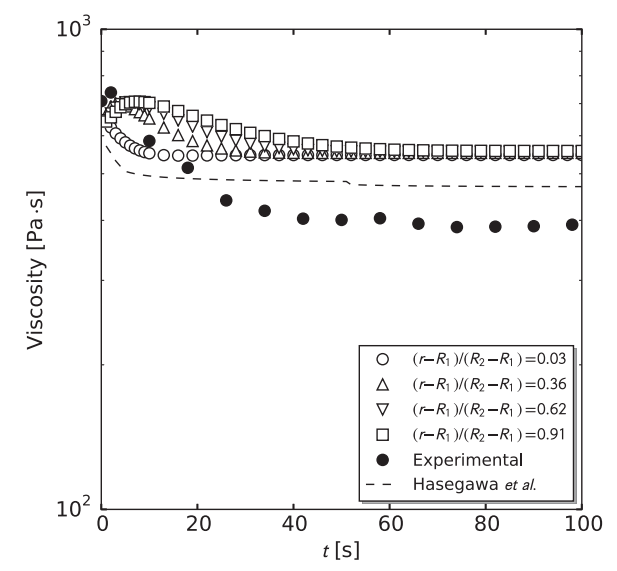

Fig. 5. Variation in viscosity as a function of time. are similar to those of the mean cluster size. This indicates that the temporary viscosity increment observed by Hasegawa is due to the temporary aggregation of particles, thereby indicating that the present model accounts for the aggregation/ dispersion behavior of particles in a uniform shear field more accurately. On the other hand, the numerical viscosity value at steady state was more than $\sim 1.4$ times the experimental value.

In the context of the results discussed thus far, we mention that particle dispersion occurs very slowly when compared with the time scale of the velocity profile, although the local mean number of particles in a cluster suddenly decreases immediately after the sudden increase in the shear rate near the inner cylindrical region for the smallest value of the bonding energy. Near the inner cylinder wall, a large shear is applied. When the bonding energy between particles is small, the large clusters break up quickly. On the other hand, the mean number of particles in a cluster remains large near the region of the outer cylinder, particularly when the bonding energy is large. In this region, the shear rate is small throughout the course of the simulation. Thus, the time dependence of the particle dispersion is considered to also depend on the strain including the history of the shear rate addition.

The local solid volume fraction did not change along the radial direction when compared with that at the initial state because the radial velocity was larger than the circumferential velocity. This indicates that the clusters were subject to advection not along the radial direction but along the circumferential direction. This is the reason for the observed viscosity variation arising due to the aggregation/breakup of cluster.

Figure 6 shows the cumulative frequencies of the clusters at $\left(r-R_{1}\right) /\left(R_{2}-R_{1}\right)=0.03,0.36,0.62$, and 0.91 for a bonding energy of $F_{0}=2.7 \times 10^{-12} \mathrm{~J}$. The solid line indicates the initial cluster size distribution as obtained by Hasegawa. The dashed and dotted lines indicate the experimental results at $t=10$ and $100 \mathrm{~s}$, respectively.

From Fig. 6(a), we observe that the cumulative frequency immediately after the shear rate change at $t=0.01 \mathrm{~s}$ is nearly equal to the initial one. At $t=0.5 \mathrm{~s}$, the number of clusters with particles greater than 20 increases. Subsequently, the cluster breaks up upon addition of high shear, and a steadystate value is reached at $t=10 \mathrm{~s}$. Upon comparing the experimental and numerical results at $t=10$ and $100 \mathrm{~s}$, we note there are smaller clusters as per the model. Overall, the simulated and experimental results show good agreement; however, the model underestimates the bonding energy of small clusters.

Figure 7 shows the time dependence of the mean cluster size for bonding energies of $F_{0}=2.7 \times 10^{-13}, 2.7 \times 10^{-12}$, and 
(a)

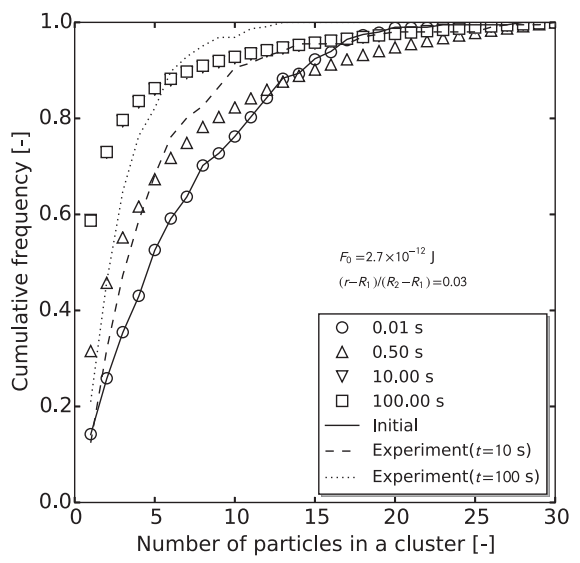

(b)

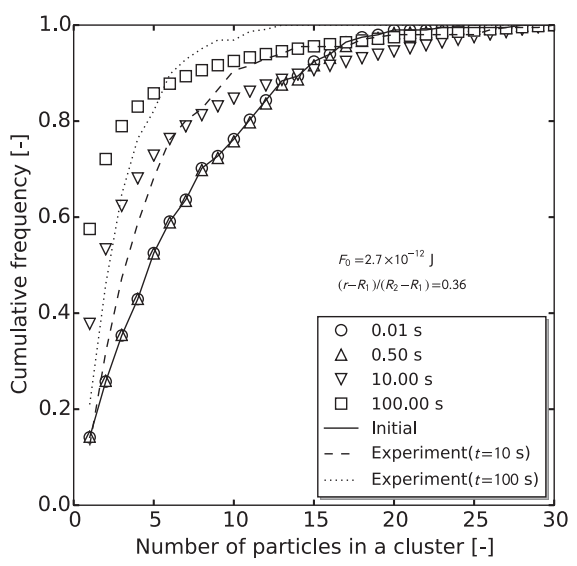

(c)

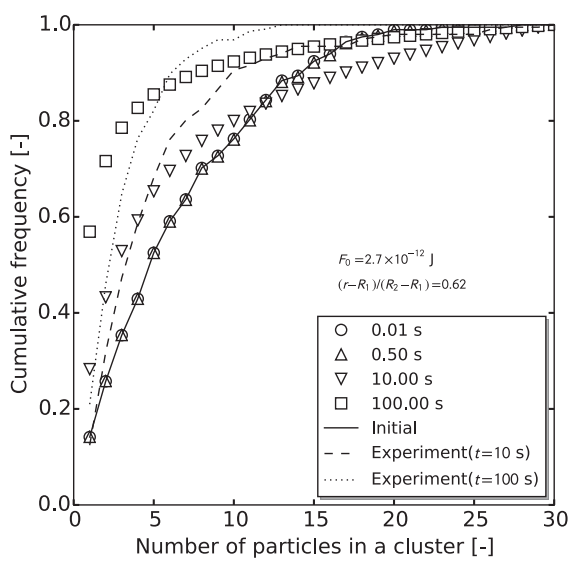

(d)

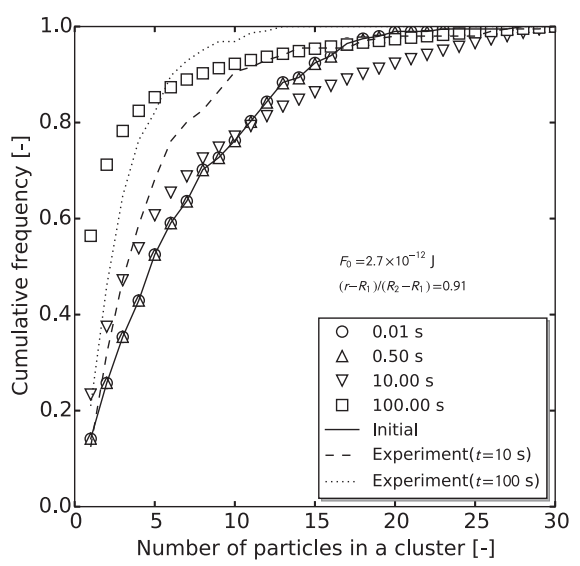

Fig. 6. Cumulative frequencies of clusters along with experimental results for $\left(r-R_{1}\right) /\left(R_{2}-R_{1}\right)=$ (a) 0.03 , (b) 0.36 , (c) 0.62 , and (d) 0.91 .
$2.7 \times 10^{-11} \mathrm{~J}$. Figure 8 shows the plot of the mean number of particles in a cluster as a function of the cumulative strain for the same bonding energies. In the case of $F_{0}=2.7 \times 10^{-11} \mathrm{~J}$, the curves of the mean cluster size vs. the strain overlap for all values of $\left(r-R_{1}\right) /\left(R_{2}-R_{1}\right)(=0.03,0.36,0.62$, and 0.91$)$. This indicates that the breakup behaviors of clusters show different time characteristics for each value of $\left(r-R_{1}\right) /\left(R_{2}-R_{1}\right)$, whereas the strain dependence of the breakup characteristics of a cluster is equal for all $\left(r-R_{1}\right) /\left(R_{2}-R_{1}\right)$ values in this case. In the other cases, in contrast, certain differences are observed at $\left(r-R_{1}\right) /\left(R_{2}-R_{1}\right)=0.03$. Near the wall region, wherein a large strain was added, the mean cluster size does not correspond to the cumulative strain. Moreover, in the case of $F_{0}=2.7 \times$ $10^{-13} \mathrm{~J}$, the mean cluster size reaches a minimum at a strain value of 10 and subsequently steadily hovers around this minimum value regardless of increase in the strain. These

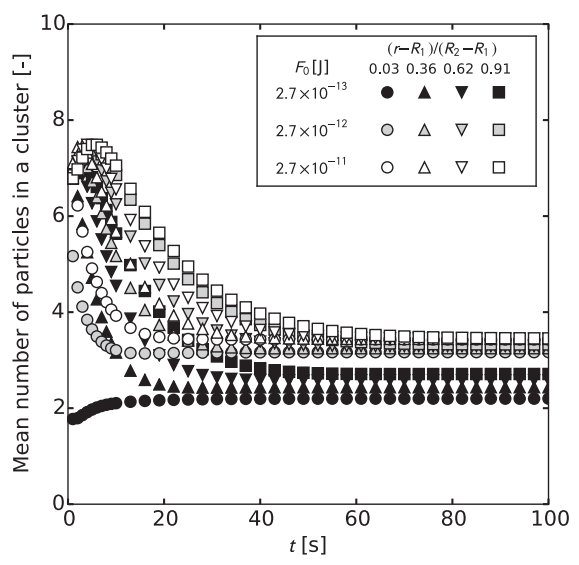

Fig. 7. Time dependence of mean cluster size for different bonding energies.

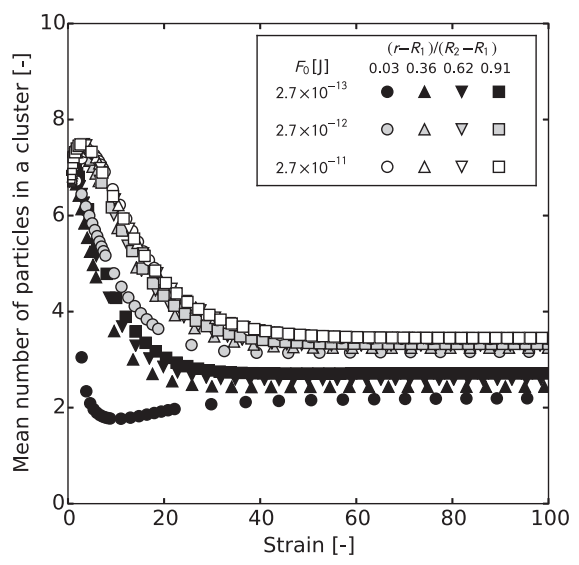

Fig. 8. Dependence of mean cluster size on applied strain for different bonding energies. 
results indicate that the local mean number of particles in a cluster is well correlated with the strain when the bonding energy is sufficiently large. Komoda et al. have reported that the strain is the primary factor affecting the time dependence of the dispersion characteristics on the overall cluster size $k_{\mathrm{m}}$. In this study, the local time dependence of the cluster size is also found to depend on the local strain for each bonding energy. Figure 9 shows the asymptotic values of the mean cluster size at $F_{0}=2.7 \times 10^{-13}, 2.7 \times 10^{-12}$, and $2.7 \times 10^{-11} \mathrm{~J}$. In the figure, the asymptotic value of the mean cluster size varies nonlinearly as the logarithmic value of $F_{0}$. This indicates that the breakup behavior of the clusters is not determined by only the bonding energy. The apparent viscosity is not linear with respect to the cluster size. The balance between the bonding energy and viscosity may determine the dispersion characteristics.

\section{CONCLUSION}

We performed two-dimensional numerical computations of unsteady flow of a suspension between two coaxial cylinders and investigated the dispersion characteristics based on a novel thixotropy model. The simulation results were compared with experimental time-variation characteristics. The effect of bonding energy between particles in the model was also considered.

From a comparison of the numerical data with experimental data, we observed that our model sufficiently accurately reproduces the time variation of the particle dispersion behavior. The velocity profile developed very quickly independent of the bonding energy, while cluster breakup occurred gradually. The cluster breakup behavior was

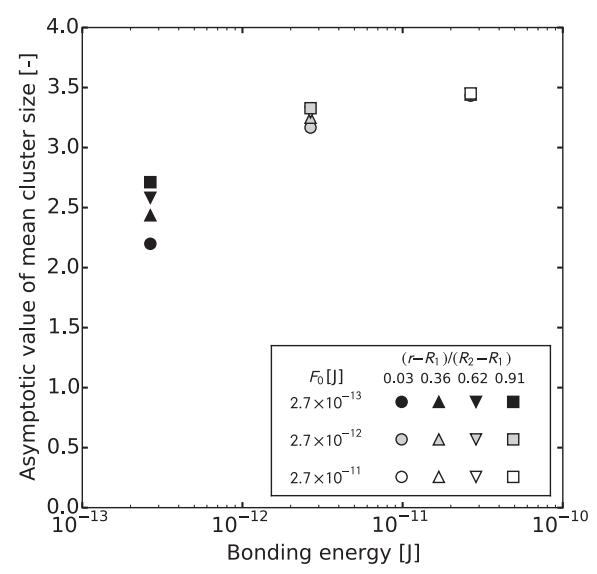

Fig. 9. Asymptotic values of mean cluster size for different bonding energies. determined by the local strain. Thus, we concluded that the local strain was the primary factor determining the dispersion characteristics. On the other hand, the asymptotic value of the mean number of particles in a cluster strongly depended on the bonding energy, but this dependency was not linear. This indicates the existence of a certain critical bonding energy with regard to the dispersion characteristics. We believe that our findings can significantly contribute to the field of polymer composites.

\section{NOTATIONS}

\begin{tabular}{|c|c|c|}
\hline$d_{\mathrm{k}}$ & $\begin{array}{l}\text { cluster size of a cluster composed of } \\
k \text { particles }\end{array}$ & $\mathrm{m}$ \\
\hline$F_{0}$ & bonding energy & $\mathrm{J}$ \\
\hline$k_{\mathrm{b}}$ & Boltzmann constant & $\mathrm{J} \cdot \mathrm{K}^{-1}$ \\
\hline$k_{\mathrm{m}}$ & mean number of particles in a cluster & - \\
\hline$N_{\mathrm{b}}$ & number of bonds to broken & - \\
\hline$n_{k}$ & $\begin{array}{l}\text { concentration of a cluster composed } \\
\text { of } k \text { particles }\end{array}$ & $\mathrm{m}^{-3}$ \\
\hline$R_{1}, R_{2}$ & radii of the inner and outer cylinders & $\mathrm{m}$ \\
\hline$r_{k}$ & $\begin{array}{l}\text { cluster radius composed of } k \\
\text { particles }\end{array}$ & $\mathrm{m}$ \\
\hline$P$ & pressure & $\mathrm{Pa}$ \\
\hline$T$ & temperature & $\mathrm{K}$ \\
\hline$t$ & time & $\mathrm{s}$ \\
\hline$u_{r}, u_{\theta}$ & radial and circumferential velocities & $\mathrm{m} \cdot \mathrm{s}^{-1}$ \\
\hline$u_{\text {wall }}$ & surface tangential velocity & $\mathrm{m} \cdot \mathrm{s}^{-1}$ \\
\hline$\alpha_{\mathrm{b}}, \alpha_{\mathrm{s}}$ & $\begin{array}{l}\text { coefficient for Brownian coagulation } \\
\text { and shear coagulation }\end{array}$ & - \\
\hline$\dot{\gamma}$ & shear rate & $\mathrm{s}^{-1}$ \\
\hline$\varepsilon, \varepsilon_{\max }$ & $\begin{array}{l}\text { void fraction and maximum void } \\
\text { fraction }\end{array}$ & - \\
\hline$\phi, \phi_{0}$ & $\begin{array}{l}\text { apparent and original solid volume } \\
\text { fraction }\end{array}$ & - \\
\hline$\phi_{\mathrm{p}, \max }$ & maximum packing fraction & - \\
\hline$\eta, \eta_{0}$ & $\begin{array}{l}\text { suspension viscosity and dispersed } \\
\text { media viscosity }\end{array}$ & $\mathrm{Pa} \cdot \mathrm{s}$ \\
\hline$\lambda(\xi)$ & computational domain cell ratio & - \\
\hline$\rho$ & density & $\mathrm{kg} \cdot \mathrm{m}^{-3}$ \\
\hline$\tau_{r r}, \tau_{r \theta}, \tau_{\theta \theta}$ & stress components & $\mathrm{Pa}$ \\
\hline
\end{tabular}




\section{REFERENCES}

1) Smoluchowski MV, Z Phys Chem, 92, 129 (1917).

2) Swift DL, Friedlander SK, J Colloid Sci, 19, 621 (1964).

3) Higashitani K, Tanaka T, Matsuno Y, J Colloid Interface Sci, 63, 551 (1978).

4) Higashitani K, Ogawa R, Hosokawa G, Matsuno Y, J Chem Eng Jpn, 15, 299 (1982).

5) Higashitani K, Iimura K, Sanda H, Chem Eng Sci, 56, 2927 (2001).

6) Usui H, Kagaku Kogaku Ronbunshu, 25, 459 (1999).

7) Usui H, Li L, Kinoshita S, Suzuki H, J Chem Eng Jpn, 34, 360 (2001).

8) Mustafa, Usui H, Shige I, Suzuki H, Kobayashi T, Miyazaki Y, Ioroi T, Yasuda K, J Chem Eng Jpn, 37, 31 (2004).

9) Hasegawa E, Suzuki H, Kameyama K, Komoda Y, Usui H, Adv Powd Tecnol, 20, 139 (2009).
10) Hasegawa E, Suzuki H, Komoda Y, Usui H, Nihon Reoroji Gakkaishi (J Soc Rheol, Jpn), 37, 191 (2009).

11) Masuda K, Suzuki H, Komoda Y, Hidema R, Nihon Reoroji Gakkaishi (J Soc Rheol, Jpn), 41, 75 (2013).

12) Masuda K, Suzuki H, Komoda Y, J Chem Eng Jpn, 46, 524 (2013).

13) Taylor GI, Philosophical Transactions of the Royal Society of London Series A, 223, 289 (1923).

14) Barbot E, Dussouillez P, Bottero JY, Moulin P, Chem Eng J, 156, 83 (2010)

15) Ouchi M, Takahashi T, Shirakashi M, Nihon Reoroji Gakkaishi (J Soc Rheol, Jpn), 35, 107 (2007).

16) Komoda Y, Kameyama K, Hasegawa E, Suzuki H, Usui H, Endo Y, Syudo A, Adv Powd Tecnol, 19, 507 (2008).

17) Simha R, J Appl Phys, 23, 1020 (1952). 